\title{
MODELO DE VALORACIÓN FINANCIERA PARA UN PRODUCTO INNOVADOR: APLICACIÓN A UN PRODUCTO DE LA UNIVERSIDAD NACIONAL DE COLOMBIA SEDE MEDELLÍN
}

\author{
Diana Milena Osorno Alzate \\ Sergio Botero Botero
}

Recibido: 21/10/2012

Aprobado: 25/10/2013

\section{RESUMEN}

Este trabajo incorpora los resultados de una investigación asociada con la gestión de la innovación y con métodos de valoración financiera, e incluye una propuesta de modelo para valorar financieramente productos innovadores existentes en la Universidad Nacional de Colombia, Sede Medellín.

Al final, se presenta el modelo aplicado a un producto innovador existente en la Universidad Nacional de Colombia, Sede Medellín.

Palabras clave: innovación, modelos de gestión de innovación, valoración, métodos de valoración financiera.

Magíster en Ingeniería Administrativa, Facultad de Minas, Universidad Nacional de Colombia, Sede Medellín. Calle 59 A 63 - 20 Bloque 19 (Autopista Norte). (4) 4309665 - 3005143487. Correo electrónico: dmosorno@unal.edu.co

** D.Sc. Profesor Asociado, Departamento de Ingeniería de la Organización, Facultad de Minas, Universidad Nacional de Colombia Sede Medellín. Carrera 80 N.`65-223.Tel (4)4255212.Correo electrónico: sbotero@unal.edu.co 


\title{
FINANCIAL VALUATION MODEL FOR AN INNOVATIVE PRODUCT: APPLICATION TO A PRODUCT OF UNIVERSIDAD NACIONAL DE COLOMBIA (MEDELLÍN CAMPUS)
}

\begin{abstract}
This work incorporates results of a research associated to innovation management and financial valuation methods; it also includes a model proposal to financially valuate innovative products existing at Universidad Nacional de Colombia (Medellin Campus).
\end{abstract}

At the end, the article shows a model applied to an innovative product existing at Universidad Nacional de Colombia (Medellin Campus).

Key words: Innovation; innovation management models; valuation; financial valuation methods. 


\section{INTRODUCCIÓN}

En Colombia, la Ley 1286 [1] aprobada por el Congreso de la República de Colombia el 23 de enero de 2009, establece como su objetivo general fortalecer el Sistema Nacional de Ciencia y Tecnología y a COLCIENCIAS para lograr un modelo productivo sustentado en la ciencia, la tecnología y la innovación, para darles valor agregado a los productos y servicios de nuestra economía, y propiciar el desarrollo productivo y una nueva industria nacional.

La Universidad Nacional de Colombia y su Sede Medellín no son indiferentes a los lineamientos nacionales y, por el contrario, orientan dentro de sus pilares fundamentales la investigación y la consolidación de grupos de investigación, la innovación, la gestión tecnológica y la transferencia del conocimiento a la sociedad. Adicionalmente, incorporan entre las líneas de acción de su documento Rector, Plan Global de Desarrollo 2010-2012 [2], la línea de ciencia, tecnología, innovación y creación artística, que busca, entre otras, la articulación de la investigación con la extensión, dada su poca visibilidad de interacción y su importante función para contribuir al desarrollo del país. En efecto, vale la pena referenciar a Abello [3], quien resalta el papel protagónico que tiene la universidad dentro del entorno científico para apoyar la innovación sustentado en la capacidad para desarrollar investigaciones científicas y tecnológicas.

Este trabajo tiene relevancia debido a que en la Institución se promueve la investigación y la transferencia de sus resultados a la empresa, pero actualmente no está definida una metodología oficial adoptada por la Universidad Nacional de Colombia, Sede Medellín, para valorar los productos innovadores con fines transferibles.

Por tanto, es relevante adelantar un modelo de valoración financiera que retome aspectos importantes de los métodos existentes en el mercado y que integre elementos particulares de la Institución para que el modelo pueda ser reconocido y validado. Para ello, en primer lugar, se presentan conceptos o elementos teóricos de innovación, valoración, gestión de innovación en Colombia, en la Universidad Nacional de Colombia y en la Sede Medellín. Seguidamente, se presentan los métodos de valoración financiera, un cuadro comparativo con sus ventajas y desventajas, y el análisis de los métodos, aplicados a la evaluación de productos innovadores. En tercer lugar, se propone un modelo de valoración financiera para la Universidad Nacional de Colombia Sede Medellín, sus variables, su comportamiento simulado y su aplicación a un producto innovador existente en la Universidad Nacional de Colombia y, finalmente, las conclusiones de la investigación.

\section{MATERIALES Y MÉTODOS}

Para obtener la propuesta de valoración financiera aplicada a uno de los productos innovadores de la Universidad Nacional de Colombia, Sede Medellín, fue necesario hacer una revisión documental de conceptos, de metodologías de valoración financiera y de productos innovadores resultantes de las investigaciones adelantadas en la Universidad; realizar entrevistas a investigadores y personas vinculadas a la institución y conocedoras del tema, así como también hacer simulaciones para conocer el comportamiento de los métodos de valoración financiera existentes en el mercado y de la propuesta de valoración.

\section{MARCOTEÓRICO}

\subsection{El concepto de innovación}

Aunque la literatura ofrece un variado conjunto de definiciones y términos relativos al concepto Innovación y a sus tipologías, se relacionan tres connotaciones importantes que cobran relevancia en este trabajo porque son explícitas al asociar el concepto con las necesidades y la introducción de los resultados al mercado:

- "La innovación es el conjunto de actividades inscritas en un determinado período de tiempo 
y lugar, que conducen a la introducción con éxito en el mercado, por primera vez, de una idea en forma de nuevos productos, servicios o técnicas de gestión y organización” [4].

- Una invención se refiere al resultado directo de las actividades de investigación, mientras que la innovación implica un producto comercial. Las innovaciones parten de la idea de un producto/proceso que ha tenido en cuenta tanto la viabilidad como la necesidad del mercado; se trata, por tanto, de un proceso [5].

- La innovación es un factor básico de desarrollo en los países avanzados, no consiste únicamente en la incorporación de tecnología, sino que ayuda a prever las necesidades de los mercados y a detectar nuevos productos, procesos y servicios de mayor calidad, generando nuevas prestaciones con el menor costo posible [6].

\subsection{El concepto de valoración}

Según Fernández [7], el valor no debe confundirse con el precio, que es la cantidad a la que el vendedor y comprador acuerdan realizar una operación de compra-venta. Afirma que algunos propósitos para una valoración son:

- Operaciones de compra-venta: Para el comprador, la valoración le indica el precio máximo a pagar; mientras que para el vendedor, la valoración le indica el precio mínimo por el que debe vender.

- Identificación de los impulsores de valor. La valoración permite identificar las fuentes de creación y destrucción de valor.

\subsection{La función exponencial natural}

Haeussler y Paul [8] exponen que uno de los números más útiles como base de una función exponencial es cierto número irracional denotado por la letra e, en honor del matemático suizo Leonardo Euler (1707-1783):

$$
e=2.71828
$$

Aunque la función exponencial natural puede parecer una base extraña, surge de manera natural en cálculo. También surge en el análisis económico y en problemas que implican crecimiento o decrecimientos naturales como estudios poblacionales, interés compuesto y decaimiento radioactivo.

\subsection{El concepto de utilidad}

En economía se llama utilidad a la capacidad que tiene una mercancía o servicio de dar satisfacción a una necesidad. En un sentido más amplio, utilidad es equivalente a bienestar, satisfacción, etc. Se dice que un bien o servicio tiene utilidad para alguien si esta persona prefiere poseerlo a no poseerlo. A partir de esta idea se construye la llamada función de utilidad que indica que la utilidad total de un individuo depende de los bienes que consume y de las cantidades consumidas de cada uno [9].

\subsection{Elementos teóricos asociados a la gestión de la innovación en Colombia, en la Universidad Nacional de Colombia y en la Sede Medellín}

En Colombia, la institucionalización de la investigación científica y tecnológica ha avanzado en los tres últimos lustros soportada por el Sistema Nacional de Ciencia y Tecnología (SNCyT), cuyas instancias de dirección y coordinación y sus principales programas y estrategias fueron establecidos por ley a comienzos de los 90, con COLCIENCIAS como su Secretaría Técnica y Administrativa. En este contexto, y como una iniciativa específica para fortalecer la comunidad científica colombiana, COLCIENCIAS viene impulsando, desde 1992, la creación y consolidación de Grupos de Investigación en universidades y centros de investigación y desarrollo tecnológico [10]. 
Por su parte, y de manera congruente con esta propuesta del SNCyT, un significativo número de universidades ha avanzado en la institucionalización de la investigación, dándole a esta actividad un lugar destacado en sus planes de desarrollo institucional y dotándola de instancias administrativas y financieras, a la vez que impulsando la creación de nuevos grupos de investigación y el fortalecimiento de los existentes. Producto de esta congruencia estratégica entre el SNCyT y las universidades, el número de grupos de investigación ha crecido vertiginosamente en el país, como puede observarse de los resultados de las convocatorias COLCIENCIAS [10].

Es por esto que las instituciones universitarias desempeñan un papel importante dentro del SNCyT por concentrar el mayor número de grupos de investigación e investigadores, por definir en la mayoría de ellas la investigación como área misional, y por ser receptoras de la mayor parte de los recursos destinados al sistema por parte del Estado colombiano. Por su complejidad institucional, la Universidad Nacional de Colombia ha estructurado su propio sistema al servicio de la investigación que está conformado por los investigadores, grupos, centros e institutos, organismos de apoyo, infraestructuras y recursos, y una normatividad apropiada para la gestión. En relación con la Universidad Nacional de Colombia Sede Medellín, es de resaltar el crecimiento de grupos y el crecimiento de número de grupos clasificados en las categorías A1 y A. Entre el año 2009 y 2010 se pasó de 5 a 9 grupos en categoría A1, y de 10 a 18 en categoría A.

\subsection{Los métodos de valoración financiera}

Existen diversas metodologías para la valoración financiera de productos en economía, administración e ingeniería. Cruz et al. [11] destacan cuatro métodos principales que son de tipo contable, de mercado, de flujo de caja descontado y de opciones reales.

\subsubsection{Métodos contables}

En el caso de la valoración de una empresa, la base de estimación es el valor de su patrimonio. A partir del valor constatado en los libros de contabilidad, se puede determinar el valor contable, el valor contable ajustado, el valor de liquidación y el valor sustancial, todos los cuales tienen por base el balance general. El valor contable es el valor -nominal- del patrimonio que figura en los libros de contabilidad y puede calcularse también como la diferencia entre el activo total y el pasivo exigible.

\subsubsection{Métodos de mercado}

Son varias las metodologías asociadas a este método: el valor del mercado, método de múltiplos, valor de reposición y valor de liquidación.

\subsubsection{Métodos de flujos de caja}

En este tipo de metodología se asume que el valor de la empresa será la suma de los flujos futuros que genere la compañía, descontados a una tasa que representa el costo del dinero. De esta forma puede observarse que para estas metodologías no es importante el precio de los activos en el mercado, sino el flujo de efectivo que los mismos puedan generar formando parte de la firma.

\subsubsection{Método de Opciones Reales}

Hernández [12], considera que las opciones reales no son más que los derechos, sin obligaciones, de realizar acciones concretas que permitan maximizar el lado favorable o limitar el lado negativo de una inversión de capital. Cruz et al. [11] definen que las opciones reales reciben denominaciones o tipos según el objeto de cada una. Los principales tipos de opciones reales son:

- Opción de aplazar o aprender. Existe cuando se puede tomar la decisión de esperar un tiempo para invertir, ya sea para obtener mayor información o porque se cree que en futuro las condiciones de mercado serán mejores. 
- Opción de salir o desinvertir. Se presenta cuando el proyecto tiene la flexibilidad necesaria para que una vez iniciado no se lleve a cabo o no se siga sin él. La opción de desinvertir se presenta cuando un proyecto se lleva a cabo por etapas, y en la finalización de cada segmento se toma de nuevo la decisión de continuar o retirarse del proyecto.

En la tabla 1 se presentan las ventajas y las desventajas que ofrece cada uno de los métodos para una posible valoración financiera de productos innovadores.

Como los métodos de valoración tienen aspectos a favor y desventajas, la pertinencia de utilizar uno u otro radica en el producto que se vaya a realizar y en la información disponible para realizar la valoración.

\subsection{Análisis de los métodos de valoración finan- ciera, aplicado a la evaluación de productos innovadores}

Por analogía con la valoración de empresas, la valoración singular de un producto con métodos contables implica considerar como valor contable o base, el valor registrado en libros al momento de su adquisición. Para el caso de un producto innovador de la Universidad Nacional de Colombia Sede Medellín, que es resultado de varios proyectos de investigación, se podría disponer de información de los presupuestos de los proyectos discriminados por rubros de inversión pero no de los elementos que integran un método contable (activos, pasivos, patrimonio). Entre los métodos de mercado, no cabe aplicar el valor de un producto nuevo entre los métodos que consideran el valor de las acciones en bolsa el cual es válido para empresas con tra-

Tabla 1. Ventajas y desventajas de los métodos en evaluación financiera

\begin{tabular}{|c|c|c|}
\hline Método & Ventajas & Desventajas \\
\hline \multirow{3}{*}{ Contables } & \multirow{3}{*}{$\begin{array}{l}\text { En la gran mayoría de los casos, es posible recopilar } \\
\text { información para determinar el valor contable en espe- } \\
\text { cial si las necesidades de información están asociadas } \\
\text { a compras, gastos, recursos de funcionamiento, etc. }\end{array}$} & $\begin{array}{l}\text { Su información no es suficiente para realizar una valora- } \\
\text { ción de productos y para determinar su precio de venta. }\end{array}$ \\
\hline & & $\begin{array}{l}\text { Para el caso de la Universidad Nacional de Colombia, la } \\
\text { información contable asociada a productos innovadores } \\
\text { no es fácilmente identificable. }\end{array}$ \\
\hline & & $\begin{array}{l}\text { No refleja lo que pasa en el mercado ni incorpora la ley } \\
\text { de oferta y demanda. }\end{array}$ \\
\hline \multirow[b]{2}{*}{ De mercado } & $\begin{array}{l}\text { La mayor parte de la información de sus parámetros } \\
\text { puede consultarse en el mercado. }\end{array}$ & $\begin{array}{l}\text { Para conocer el valor de los parámetros es necesario hacer } \\
\text { algún tipo de estudio de mercado. }\end{array}$ \\
\hline & $\begin{array}{l}\text { Cuando el producto es similar a otros ya existentes en el } \\
\text { mercado, el valor del producto puede estar referenciado } \\
\text { por el valor de otro producto. }\end{array}$ & $\begin{array}{l}\text { El valor de mercado no tiene en cuenta la productividad } \\
\text { del activo en el largo plazo. }\end{array}$ \\
\hline \multirow{3}{*}{$\begin{array}{l}\text { De flujo de } \\
\text { caja }\end{array}$} & Son los métodos más aceptados y probados en el medio. & $\begin{array}{l}\text { Para la valoración requiere información contable y de } \\
\text { mercado. }\end{array}$ \\
\hline & $\begin{array}{l}\text { Además de considerar el valor de mercado del producto, } \\
\text { ajusta ese valor según las ganancias que se espera a } \\
\text { futuro. }\end{array}$ & $\begin{array}{l}\text { Requiere elaborar la información contable del producto } \\
\text { (flujos de caja) y consultar información en el mercado. }\end{array}$ \\
\hline & Reconocido y aceptado en el mercado financiero. & \\
\hline \multirow{2}{*}{$\begin{array}{l}\text { De opciones } \\
\text { reales }\end{array}$} & $\begin{array}{l}\text { Contiene más parámetros de información que los demás } \\
\text { métodos buscando obtener una estimación que permita } \\
\text { acercarse a la realidad. }\end{array}$ & $\begin{array}{l}\text { No todos los parámetros de cálculo son adaptables a pro- } \\
\text { ductos innovadores que salen por primera vez al mercado. }\end{array}$ \\
\hline & $\begin{array}{l}\text { Conocido y aplicado para negociaciones en el mercado } \\
\text { bursátil. }\end{array}$ & \\
\hline
\end{tabular}


yectoria en el mercado bursátil. En teoría, podría determinarse o adaptarse un método de múltiplos para productos nuevos en el mercado (en este caso, si se considera que el producto innovador es un producto mejorado de otro existente).

No es directa la aplicación de los métodos de flujo de caja por las variables que intervienen en el procedimiento de cálculo. Aunque se considera el mejor método de valoración de empresas, para aplicarlo a la valoración de productos innovadores sería necesario seleccionar y adaptar algunos conceptos según la información disponible en la institución en la que se valoraría el producto. Un concepto importante de los métodos de flujo de caja es que consideran un valor actual del producto por una expectativa futura de beneficios y un valor pasado de inversión a precios actuales. Los métodos de opciones reales son aplicables a la valoración de un producto innovador en la medida que este sea considerado un activo o una inversión productiva y se disponga de información adicional de su posible comportamiento en el mercado para determinar y valorar las opciones. Aun disponiendo de esta información, es necesario contar con un valor inicial del producto, sea contable, estimado por precios de mercado de productos similares o por flujo de caja descontado o por el precio inicial que se quiera asignar.

\section{RESULTADOS}

\subsection{Variables del método compuesto}

\subsubsection{Variables del método compuesto asociada al método de flujo de caja}

$I_{0}$ : Inversión realizada al producto a precios de hoy. Esta variable incorpora elementos de la teoría del método de flujo de caja. Para poder calcularlo, es necesario conocer los valores de tres variables. La primera, está constituida por los costos totales de las inversiones valorados en el tiempo y equivalentes al valor presente de cada una de los proyectos
(VP); la segunda, por los períodos de inversión en los cuales fueron ejecutados los proyectos (n) y la tercera, es la tasa de interés (i) que se incorpore en la fórmula. Dado lo anterior, al momento de calcular $I_{0}$, es posible disponer de varios VP cada uno con un valor diferente de $n$.

\subsubsection{Variable del método compuesto asociada al método de mercado}

Esta propuesta de modelo incorpora como referencia el método de múltiplos por considerar que un múltiplo propio de la Institución da "especificidad" al modelo de valoración y permite incorporar elementos importantes y propios para la valoración de los productos de la empresa que lo está adoptando: pública, con fines sociales y con misión investigadora.

Para la obtención del múltiplo, se procedió de la siguiente manera:

- Identificación de elementos importantes. Se identifican teniendo como referencia las definiciones de innovación, los modelos de gestión de innovación y el contexto universitario e institucional.

- Definición de las siguientes condiciones que debe cumplir el múltiplo:

- Nunca puede tomar el valor de cero (0).

- Debe estar en función de elementos importantes identificados.

- Debe permitir incluir variables que ponderan la importancia de los elementos.

- Si fuese necesario, el múltiplo debe permitir la incorporación de nuevos elementos y nuevas variables.

- Debe representar, al momento de la valoración, "utilidad" para la institución, debido a que representa la posibilidad de obtener "algún beneficio" de transferir un producto innovador al mercado. 
- Validación de los elementos y de las condiciones del múltiplo. De acuerdo con Giraldo [13], una fórmula válida para incorporar un múltiplo con las condiciones adecuadas es la función de utilidad exponencial, puesto que cumple las condiciones anteriormente descritas. En consecuencia, un múltiplo para la fórmula del método compuesto que cumple las condiciones mencionadas y que representa una función de utilidad exponencial es:

$$
e^{b_{1} \times I+b_{2} \times R+b_{3} \times U}
$$

Del múltiplo expuesto, se resalta:

- Independientemente del valor que se obtenga en el exponente, siempre se podrá valorar el producto innovador debido a que el mínimo valor del múltiplo será 1 y en ese caso el valor del producto innovador será igual a $I_{0}$.

- Contempla elementos importantes que permiten determinar "con identidad institucional" el valor del múltiplo:

- Nivel de innovación del producto (I), es decir, si el mejorado o nuevo. Como se pueden tener dos resultados posibles (mejorado o nuevo), la utilidad podrá tomar dos valores posibles: cero si es mejorado y uno si es nuevo.

- Riesgo de incorporación en el mercado (R): al momento de la valoración, el producto es o no, demandado en el mercado. En este caso, el riesgo es no disponer de mercado para el resultado de la investigación. Como se puede tener dos resultados posibles, se considerará cero si no hay mercado y uno cuando el mercado exista y sea conocido.

- Uso del producto o del proceso innovador (U): social o comercial. Este elemento tiene una relación directa con la misión institucional. Tiene dos resultados posibles: fines sociales o comerciales. Se considerará un valor de cero cuando el producto innovador esté relacionado con usos sociales y uno cuando su uso sea comercial.

- Incorpora conceptos de la función de utilidad exponencial.

- Cumple las propiedades de la función exponencial natural.

- Contiene valores $b_{1}, b_{2}$ y $b_{3}$ que corresponden a los pesos porcentuales que representan la importancia de cada uno de los elementos $I$, $R, U$. Por ser valores porcentuales, la suma de $b_{1}, b_{2}$ y $b_{3}$ debe ser igual al $100 \%$.

- Aunque la propuesta del múltiplo en el método compuesto se hace incorporando tres elementos importantes en función de la valoración del producto innovador, el múltiplo permite incorporar nuevos elementos que pueden ser relevantes para la Institución y para el evaluador. En caso que se requiera incorporar nuevos elementos de evaluación puede hacerse, siempre y cuando se tenga en cuenta que debe existir igual número de valores de $b$ como de elementos y que siempre la sumatoria de los valores de $b$ debe ser igual al $100 \%$.

\subsection{Modelo de valoración financiera: método compuesto}

La propuesta de valoración financiera es un método compuesto que incorpora referencias importantes de dos métodos de valoración: el de flujo de caja y el de mercado (múltiplo). La construcción matemática del método compuesto es:

$$
V P I(I, R, U)=I_{0} e^{b_{1} \times I+b_{2} \times R+b_{3} \times U}
$$

Donde:

VPI (I,R,U): Valor del producto innovador (para productos innovadores en la Universidad Nacional de Colombia) en función de los elementos: 
Nivel de innovación del producto (I), Riesgo de incorporación en el mercado $(\mathrm{R})$ y Uso del producto o del proceso innovador (U)

$I_{0}$ : $\quad$ Inversión realizada al producto a precios de hoy.

$$
e^{b_{1} \times I+b_{2} \times R+b_{3} \times U}: \text { múltiplo. }
$$

\subsubsection{Simulación del método compuesto}

En el figura 1 se simulan valores teóricos para 21 productos, considerando que la inversión realizada al producto al momento de la valoración es de 100 (y se mantiene constante) y variando el valor del múltiplo un rango de 1 a 2.71828 .

La variación del múltiplo se hace teniendo como referencia que el valor mínimo que puede alcanzar el múltiplo es cuando el producto es mejorado, sin mercado y para uso social. En este caso el exponente $b_{1}{ }^{*} I+b_{2}{ }^{*} R+b_{3}{ }^{*} U$ equivale a cero (0). Por otro lado, se considera que el valor máximo que puede alcanzar el múltiplo es cuando el producto es nuevo, con mercado y para uso comercial. En este caso el exponente $b_{1}{ }^{*} I+b_{2}{ }^{*} R+b_{3}{ }^{*} U$ equivale a uno (1).

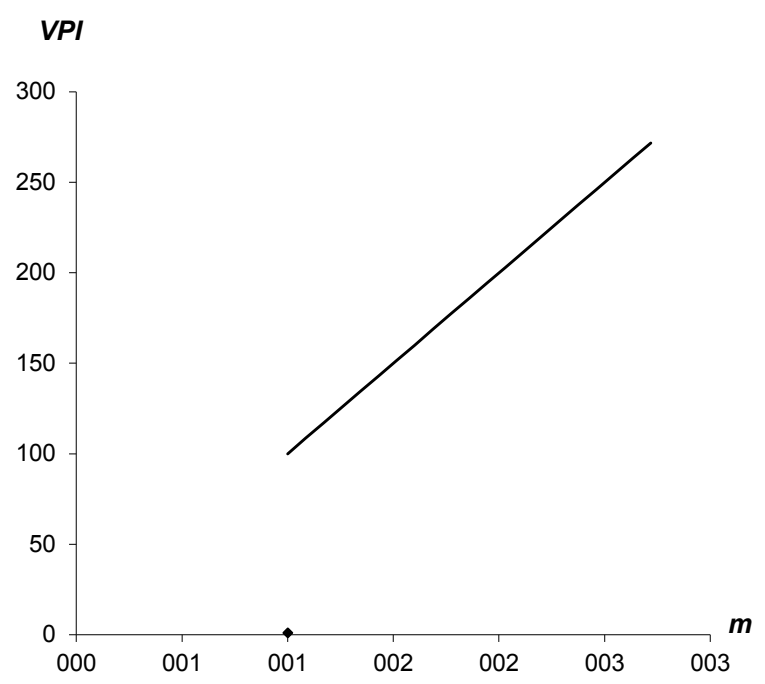

Figura 1. Valoración de un producto innovador en función de su múltiplo.

Fuente: elaboración propia.
La tendencia que presenta el valor del producto innovador cuando es fijo el valor de la inversión al momento de la valoración $\left(\mathrm{I}_{\mathrm{o}}\right)$ y el múltiplo varía, es $\left(e^{b_{1} \times I+b_{2} \times R+b_{3} \times U}\right)$ en dirección creciente.

\subsubsection{Aplicación a un caso práctico}

Para validar el modelo propuesto, se tomó información del producto innovador "Gasificador combustor", el cual ha sido resultado de tres proyectos de investigación financiados por instituciones públicas y por la empresa privada.

Por cuestiones de confidencialidad institucional no se presentan datos reales, pero con la siguiente información y los siguientes valores, se puede obtener el valor del producto:

- Los proyectos se iniciaron en los años 1995, 2000 y 2006 y tuvieron una duración de cuatro años, el primero, y tres años, los dos siguientes.

- La inversión total de los proyectos de investigación es de 776 millones de pesos.

- La inversión asociada directamente al producto innovador corresponde a 233 millones de pesos.

- El valor de la inversión al año 2011 equivalente a VF(proyecto 1$)+\mathrm{VF}$ (proyecto 2) + $\mathrm{VF}$ (proyecto 3) es de 323 millones de pesos.

- El Gasificador Combustor es considerado un producto nuevo, con mercado y con usos comerciales.

- Las variables que determinan el peso porcentual de cada uno de los elementos tienen el mismo valor, y es equivalente a 0.3333 .

Al incorporar los valores en la formula, se determina el siguiente valor para el producto innovador:

$$
\begin{aligned}
& V P I(I, R, U)=I_{0} * e^{b_{1} \times I+b_{2} \times R+b_{3} \times U} \\
& V P I(I, R, U)=393 * e^{0,33 \times 1+0,33 \times 1+0,33 \times 1} \\
& V P I(I, R, U)=1.067
\end{aligned}
$$




\section{CONCLUSIONES}

Aunque son diversas las definiciones referentes a la innovación y todas tienen su relevancia en el tiempo, es importante resaltar que en las definiciones se han incorporado elementos importantes para la innovación como introducción con éxito en el mercado [7], producto comercial [2] y necesidades de los mercados [8]. Estos nuevos elementos representan en cierta medida una evidencia de la pertinencia de este documento.

Retomando el concepto de valoración de Fernández [9], en el que "el valor no debe confundirse con el precio", la valoración financiera de un producto innovador resultante del modelo propuesto en este documento puede considerarse un valor de referencia para iniciar negociaciones. El resultado de las negociaciones que permitirá la obtención del precio del producto se obtendrá bajo los conceptos de la teoría económica relacionados con las leyes de oferta y demanda.

Son diversas las metodologías existentes para la valoración de empresas o de productos, cada una de ellas con debilidades y fortalezas particulares que deben ser consideradas por quien seleccione una metodología con el objetivo de hacer una valoración. La selección de la metodología debe hacerse teniendo en cuenta el objetivo de la valoración y la información disponible para llevarla a cabo.

No existe un modelo de valoración financiera que esté relacionado directamente con la determinación del valor de un producto innovador. En este sentido, para valorar un producto innovador en una organización (para nuestro caso, en la Universidad Nacional de Colombia Sede Medellín) es posible retomar de los modelos de valoración existentes y aplicables a las empresas y a los productos, elementos importantes de los cuales se pueda obtener información financiera en el interior de la compañía.

El método compuesto como propuesta de valoración financiera para los productos de la
Universidad Nacional de Colombia, Sede Medellín, incorpora elementos importantes de dos métodos de valoración: el de flujo de caja y el de mercado. La variable asociada al método de mercado es propia para la Institución porque integra elementos relevantes obtenidos de los conceptos de innovación, cumple condiciones establecidas para la obtención de la valoración e incorpora conceptos de la función de utilidad exponencial. Esta variable está formulada de manera que permite incluir, si fuese necesario, nuevos elementos importantes para la institución y también, variables ponderadoras.

\section{REFERENCIAS}

[1] Colombia. Congreso de la república. Ley 1286 de 2009 "Por la cual se modifica la Ley 29 de 1990, se transforma a Colciencias en Departamento Administrativo, se fortalece el Sistema Nacional de Ciencia, Tecnología e Innovación en Colombia y se dictan otras disposiciones". Bogotá: Diario Oficial No. 47.241, 2009. Artículo 1.

[2] Universidad Nacional de Colombia. Plan Global de Desarrollo 2010-2012, Por una Universidad de excelencia, investigadora, innovadora y a la vanguardia del país. Bogotá: 2009.

[3] R. Abello, "La Universidad: un factor clave para la innovación tecnológica empresarial”, Pensamiento y Gestión, N 16, pp. 28-42, 2004.

[4] J. Pavon. R. Goodman, "Proyecto Modeltec. La planificación del desarrollo tecnológico”, CDTI-CSIC, Madrid, 1981.

[5] W.G. Biemans, "Managing Innovation within Networks”, Routledge, Londres/New York, 1992.

[6] H. Aranda Gutiérrez. M. L. De La Fuente Martínez. M. N. Becerra Reza, "Propuesta metodológica para evaluar la gestión de la innovación Tecnológica (GIT) en pequeñas y medianas empresas (PYMES)" Revista Mexicana de Agronegocios, vol. XIV, n. ${ }^{\circ}$ 26, pp. 226-238, 2010.

[7] P. Fernández, Valoración de empresas ¿Cómo medir y gestionar la creación de valor? Barcelona: Ediciones Gestión S. A., 2000, 932 p.

[8] E. Haeussler. R.S. Paul . Matemáticas para la Administración y la Economía. México. Prentice Hall. 2003. 973 p.

[9] C. Sabino, Diccionario de Economía y Finanzas. Venezuela. Editorial Panapo. 1991. 301 p. 
[10] J. Robledo, "De los grupos consolidados de investigación a los sistemas dinámicos de innovación: El desafío actual del desarrollo científico y tecnológico colombiano", Dyna, vol. 74, n..$^{\circ} 152$, pp. 1-7. 2007.

[11] S. Cruz. J. Villareal. J. Rosillo. Finanzas corporativas: Valoración, Politica de Financiamiento y Riesgo. México: Internacional Thomson Editores, 2003, 636 p.
[12] M. Hernández. "Aplicación de la teoría de opciones reales en un contexto de globalización financiera", InterSedes: Revista de las Sedes Regionales, vol. VIII, n. ${ }^{\circ}$ 14. pp. 65-73, 2007.

[13] Entrevista con Norman Diego Giraldo Gómez, Profesor asociado en dedicación exclusiva de la Escuela de Estadística de la Universidad Nacional de Colombia, Medellín, 12 de abril de 2011. 